\title{
Orally administered betaine reduces photodamage caused by UVB irradiation through the regulation of matrix metalloproteinase-9 activity in hairless mice
}

\author{
A-RANG IM ${ }^{1}$, HEE JEONG LEE ${ }^{1}$, UI JOUNG YOUN ${ }^{2}$, JIN WON HYUN ${ }^{3}$ and SUNGWOOK CHAE ${ }^{1,4}$ \\ ${ }^{1}$ KM-Based Herbal Drug Development Group, Korea Institute of Oriental Medicine, Daejeon 305-811, Republic of Korea; \\ ${ }^{2}$ Department of Pharmaceutical Sciences, Daniel K Inouye College of Pharmacy, University of Hawaii at Hilo, Hilo, \\ HI 96720, USA; ${ }^{3}$ School of Medicine and Institute for Nuclear Science and Technology, Jeju National University, \\ Jeju 690-756; ${ }^{4}$ Korean Medicine Life Science, University of Science and Technology, \\ Daejeon 305-333, Republic of Korea
}

Received January 7, 2015; Accepted October 19, 2015

DOI: $10.3892 / \mathrm{mmr} .2015 .4613$

\begin{abstract}
Betaine is widely distributed in plants, microorganisms, in several types of food and in medical herbs, including Lycium chinense. The administration of $100 \mathrm{mg}$ betaine $/ \mathrm{kg}$ body weight/day is an effective strategy for preventing ultraviolet irradiation-induced skin damage. The present study aimed to determine the preventive effects of betaine on ultraviolet $B(U V B)$ irradiation-induced skin damage in hairless mice. The mice were divided into three groups: Control $(n=5)$, UVB-treated vehicle $(n=5)$ and UVB-treated betaine $(n=5)$ groups. The level of irradiation was progressively increased between $60 \mathrm{~mJ} / \mathrm{cm}^{2}$ per exposure at week 1 (one minimal erythematous dose $=60 \mathrm{~mJ} / \mathrm{cm}^{2}$ ) and $90 \mathrm{~mJ} / \mathrm{cm}^{2}$ per exposure at week 7 . The formation of wrinkles significantly increased following UVB exposure in the UVB-treated vehicle group. However, treatment with betaine suppressed UVB-induced wrinkle formation, as determined by the mean length, mean depth, number, epidermal thickness and collagen damage. Furthermore, oral administration of betaine also inhibited the UVB-induced expression of mitogen-activated protein kinase kinase (MEK), extracellular signal-regulated kinase (ERK), and matrix metalloproteinase-9 (MMP-9). These findings suggested that betaine inhibits UVB-induced skin damage by suppressing increased expression of MMP-9 through the inhibition of MEK and ERK.
\end{abstract}

Correspondence to: Dr Sungwook Chae, KM-Based Herbal Drug Development Group, Korea Institute of Oriental Medicine, 1672 Yuseongdae-ro, Yuseong-gu, Daejeon 305-811, Republic of Korea

E-mail: kendall@kiom.re.kr

Key words: photoaging, wrinkles, ultraviolet $\mathrm{B}$, betaine, matrix metalloproteinase-9

\section{Introduction}

Skin aging is a complex biological process, which is affected by a combination of endogenous/intrinsic and extrinsic factors. Intrinsic aging involves genetics, cellular metabolism, hormones and metabolic processes, whereas extrinsic aging is affected by chronic light exposure, pollution, ionizing radiation, chemicals and toxins (1). Extrinsic skin aging is characterized by elastosis in the upper dermis, destruction of fibrillar structure, augmentation of intercellular substances and moderate infiltration of inflammatory mediators (2).

Extrinsic aging is primarily associated with exposure to environmental factors, including ultraviolet (UV) irradiation. Chronic UV irradiation damages skin proteins and induces wrinkle formation, dryness, roughness, shallowness and histological changes in humans and animals $(3,4)$. Furthermore, chronic UV irradiation can result in edema, erythema, inflammation, hyperpigmentation, hyperplasia and photoaging (5). Certain age-associated skin lesions have also been associated with UV irradiation, including actinic keratosis and non-melanoma skin cancer, including basal cell carcinoma and squamous cell carcinoma $(2,6)$. These pathogenic changes may be associated with UV irradiation-induced dermal alterations, including the excessive secretion of matrix metalloproteinases (MMPs), which degrade collagen and other extracellular matrix proteins (7).

Collagen is an important extracellular component in the skin and is comprised of a highly repetitive sequence of glycines (8). Dermal collagen is a major component of the skin dermis and is required to maintain skin structure; with type I collagen being the most abundant subtype of collagen found in the dermis (8). UV irradiation-induced abnormalities in the metabolism of skin collagen are the predominant causes of skin photoaging (9). The UV-induced reduction of type I collagen in the dermis is widely considered the primary cause of the wrinkled appearance observed in skin photoaging (9).

MMP-9 is a 92-kDa gelatinase, which degrades collagen IV, and is one of the primary components of the basement 
membrane (10). Mitogen-activated protein kinases (MAPKs), including extracellular signal-regulated kinase (ERK), c-Jun $\mathrm{N}$-terminal kinase and p38, are known to be involved in the regulation of MMP-9 transcription (11). Increased expression of MMP-9, resulting from the activation of specific transcription factors, has been shown to degrade collagen and elastin in the skin (11), potentially contributing to the effects of UV-induced skin damage and photoaging.

Betaine is a naturally occurring compound, which is widely distributed in plants, microorganisms, several types of food and medicinal herbs, including Lycium chinense, which has been demonstrated to have high levels of betaine (12). Notably, betaine has been reported to be beneficial for a number of conditions and diseases, including heart and liver disease $(13,14)$. In the course of screening photoprotective agents based on antioxidant activity, our previous investigation evaluated whether betaine, exhibiting antioxidant properties, may be applied for photoprotection, regardless of known traditional medicines (15). However, whether betaine has protective effects on UV-induced skin damage and photoaging remains to be fully elucidated.

In the present study, male HR-1 hairless mice were used to assess the therapeutic effects of betaine on photoaging by evaluating various parameters of photoaging following exposure to UVB irradiation. The aim of the investigation was to determine whether betaine exhibits a protective effect on UVB-induced skin damage.

\section{Materials and methods}

Materials. Betaine was purchased from Wako Pure Chemical Industries, Ltd. (Tokyo, Japan). A total of 18 HR-1 hairless male mice (6 weeks-old) were purchased from Japan SLC, Inc. (Shizuoka, Japan). UVB irradiation was induced using a UVM-225D Mineralight UV Display Lamp (UVP, LLC, Phoenix, AZ, USA). Replicas of mouse dorsal skin were obtained using a Repliflo Cartridge kit (CuDerm Corporation, Dallas, TX, USA). Antibodies targeting MMP-9 (92 kDa), phosphorylated (p)-MAPK kinase (p-MEK; $45 \mathrm{kDa}$ ), MEK (45 kDa), pERK 1/2 (42,44 kDa), and ERK 1/2 (42, 44 kDa) were purchased from Cell Signaling Technology, Inc. (Danvers, MA, USA). Secondary antibodies were purchased from Santa Cruz Biotechnology, Inc. (Santa Cruz, CA, USA).

Experimental animals. HR-1 hairless male mice were allowed to acclimate for one week prior to study. All experimental protocols were approved by the Korea Institute of Oriental Medicine Institutional Animal Care and Use Committee (11-061; Daejeon, Korea). The animals were housed under constant temperature $\left(24^{\circ} \mathrm{C}\right)$ in an atmosphere containing $50 \%$ relative humidity with a $12 \mathrm{~h}$ light/dark cycle, and were provided with access to food and water ad libitum. The mice were divided into the following three groups: Control $(n=5)$, UVB-treated vehicle $(n=5)$ and UVB-treated betaine $(n=5)$. The mice in the UVB-treated vehicle and UVB-treated betaine groups were exposed to UV irradiation, as described below, whereas the control animals were not exposed to irradiation. The mice in the UVB-treated betaine group were administered orally with $0.1 \mathrm{ml}$ water containing $100 \mathrm{mg}$ betaine $/ \mathrm{kg}$ body weight/day. As controls, the mice in the UVB-treated vehicle group were provided with drinking water only, whereas animals in the control group received no administration

$U V B$ irradiation. Mice were subjected to UVB irradiation using a UVM-225D Mineralight UV Display Lamp, which emitted radiation at a wavelength of $302 \mathrm{~nm}$. The UV strength was measured using an HD2102-2 UV meter (Delta Ohm, Padova, Italy). For the in vivo experiments, UVB radiation was applied to the backs of the mice three times each week (Monday, Wednesday, Friday) for 12 weeks. The level of irradiation was progressively increased between $60 \mathrm{~mJ} / \mathrm{cm}^{2} /$ exposure at week 1 (one minimal erythematous dose $=60 \mathrm{~mJ} / \mathrm{cm}^{2}$ ) and $90 \mathrm{~mJ} / \mathrm{cm}^{2} /$ exposure at week 7 .

Generation of skin replicas and image analysis. Replicas of mouse dorsal skin were obtained using a Repliflo Cartridge kit. Viscous spreadable fluid resin was applied to the skin surface. After drying and hardening, a solid replica was obtained from the skin. Wrinkle shadows from the impression replicas were produced by illuminating the replica on a horizontal stand with a light source angle of $35^{\circ}$, and images were recorded and analyzed using Skin Visiometer VL 650 software (Courage + Khazaka Electronic GmbH, Cologne, Germany). The parameters used for the assessment of skin wrinkles included the average length, depth and number of wrinkles.

Histological examination. Mice were anesthetized with intraperitoneal administration of a diluted solution (1:4 in phosphate-buffered saline) composed of a 2:1 mixture of $30 \mathrm{mg} / \mathrm{kg}$ Zoletil (Virbac, Carros, France) and $10 \mathrm{mg} / \mathrm{kg}$ Rompun (Bayer, Leverkusen, Germany). Following anesthesia, the middle of the dorsal skin was fixed in $10 \%$ neutral buffered formalin (Sigma-Aldrich, St. Louis, MO, USA), embedded in paraffin (Leica, Vienna, Austria) and sectioned at $5 \mu \mathrm{m}$ thickness. The sections were stained with hematoxylin and eosin (H\&E; YD Diagnostics Corp. Kyunggi-Do, Korea), and Masson's trichrome staining (YD Diagnostics Corp.) was used for collagen fiber analysis. The thickness of the epidermis was measured under a light microscope using an eyepiece micrometer (AX-70; Olympus Corporation, Tokyo, Japan).

Western blot analysis. Proteins were extracted from the skin tissue samples using a Precellys 24 homogenization system (Bertin Technologies, Montigny-le-Bretonneux, France). Total protein concentration was determined using a $\mathrm{DC}^{\mathrm{TM}}$ Protein Assay (Bio-Rad Laboratories, Inc., Hercules, CA, USA) with $2 \mathrm{mg} / \mathrm{ml}$ bovine serum albumin standard ampules (Thermo Fisher Scientific, Inc., Loughborough, UK). as a standard. A total of $20 \mu \mathrm{g}$ protein from each sample was electrophoresed on 12\% SDS-PAGE (Bio-Rad Laboratories, Inc.). The proteins were then transferred to polyvinylidene difluoride membranes (Bio-Rad Laboratories, Inc.), and the membranes were blocked for $1 \mathrm{~h}$ at $25^{\circ} \mathrm{C}$ in $5 \%$ skimmed milk. The blots were then incubated overnight at $4^{\circ} \mathrm{C}$ with the appropriate monoclonal antibodies diluted to 1:1,000: Anti-p-MEK 1/2 (polyclonal; cat. no. 9121), anti-MEK 1/2 (polyclonal; cat. no. 9122), anti-p-ERK 1/2 (polyclonal; cat. no. 9101), anti-ERK 1/2 (polyclonal; cat. no. 9102), anti-MMP-9 (polyclonal; cat. no. 3852) (all from Cell 
A

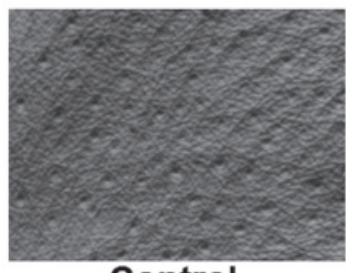

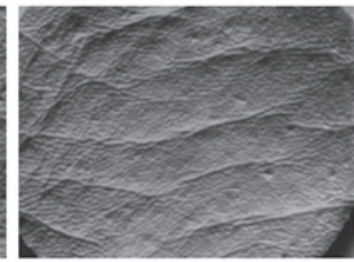

Vehicle

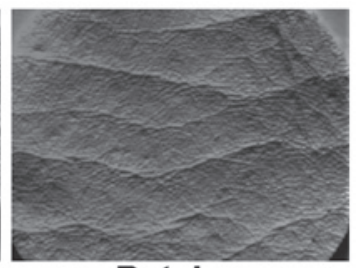

Betaine

UVB

B

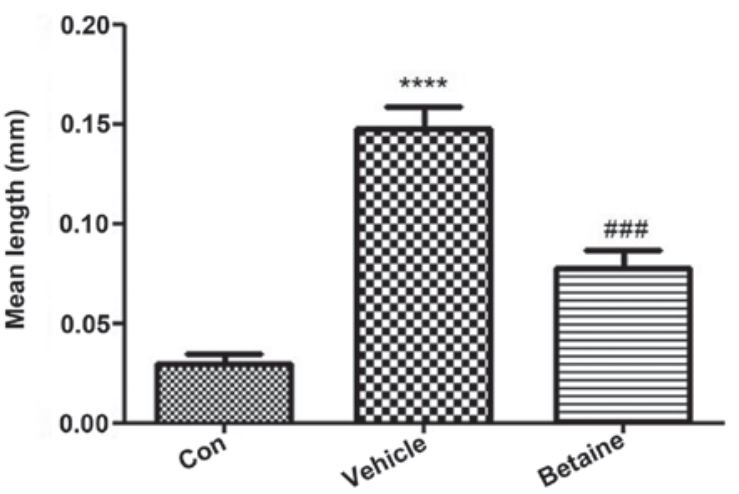

C

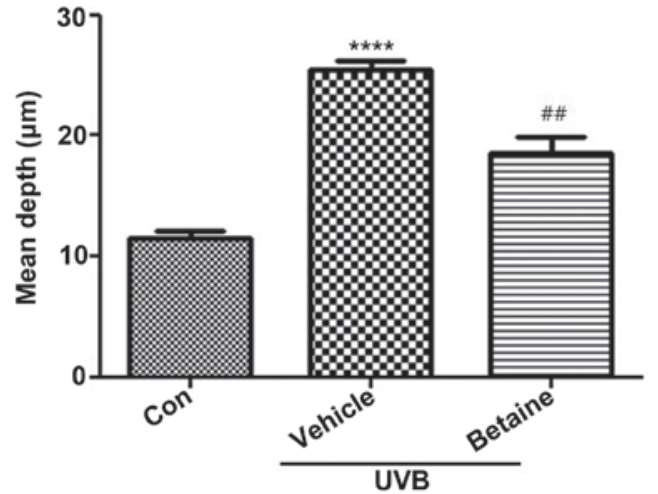

D

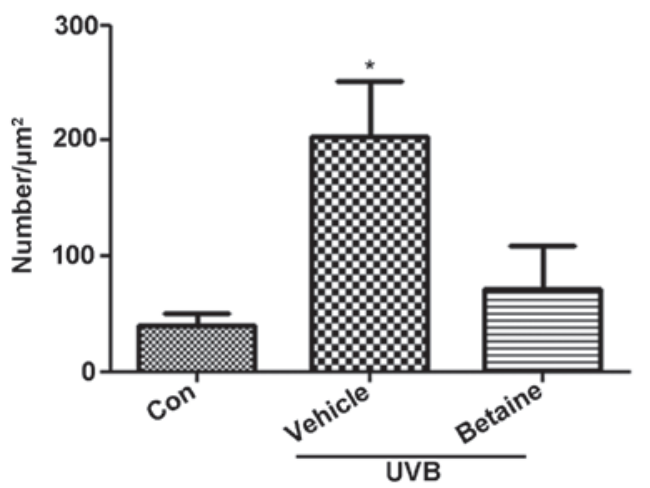

Figure 1. Betaine reduces UVB-induced wrinkle formation. Analysis of wrinkles using skin replicas obtained from the dorsal skin. The skin replicas of the control group $(n=5)$, the UVB-treated vehicle $(n=5)$ and the UVB-treated betaine $(n=5)$ group were generated at the end of the experiment. (A) Representative images of the skin replicas. Analysis of the replica images was performed using a Skin-Visiometer and its software, which allowed the determination of the (B) mean length, (C) depth and (D) number of wrinkles. Original magnification, $\mathrm{x} 200$. ${ }^{*} \mathrm{P}<0.05$ and ${ }^{* * * * *} \mathrm{P}<0.0001$, vs. Con. ${ }^{\# \# \#} \mathrm{P}<0.001$ and ${ }^{\# \#} \mathrm{P}<0.01$ vs. $\mathrm{UVB} / \mathrm{vehicle} \mathrm{UVB}$, ultraviolet B; Con, control.

Signaling Technology, Inc.) and anti- $\beta$-actin (polyclonal; cat. no. sc1616; Santa Cruz Biotechnology, Inc.). The blots were washed three times for $10 \mathrm{~min}$ each with Tris-buffered saline containing 1\% Tween-20 (Bio-Rad Laboratories, Inc.). The membranes were then incubated for $2 \mathrm{~h}$ at room temperature with anti-rabbit and anti-goat secondary antibodies (Santa Cruz Biotechnology, Inc.). The proteins were detected using enhanced chemiluminescence reagents (Bio-Rad Laboratories, Inc.). Images were captured and analyzed using ImageQuant LAS 4000 Multi Gauge software (Fuji Photo Film Co., Ltd., Tokyo, Japan).

Statistical analysis. All data are expressed as the mean \pm standard error of the mean of at least three independent experiments. 


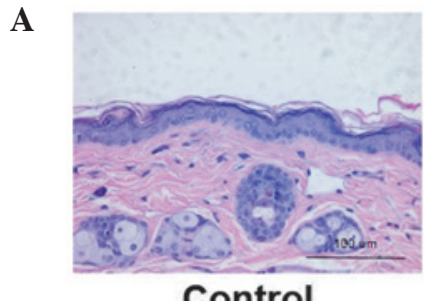

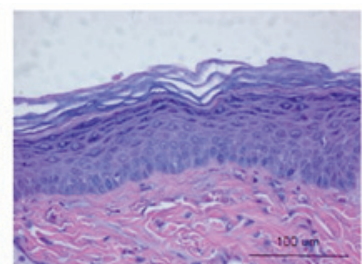

Vehicle

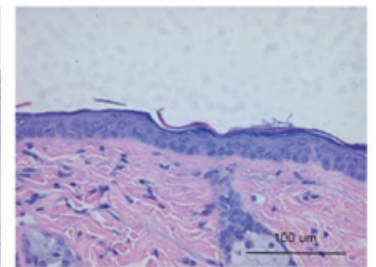

Betaine

UVB

B

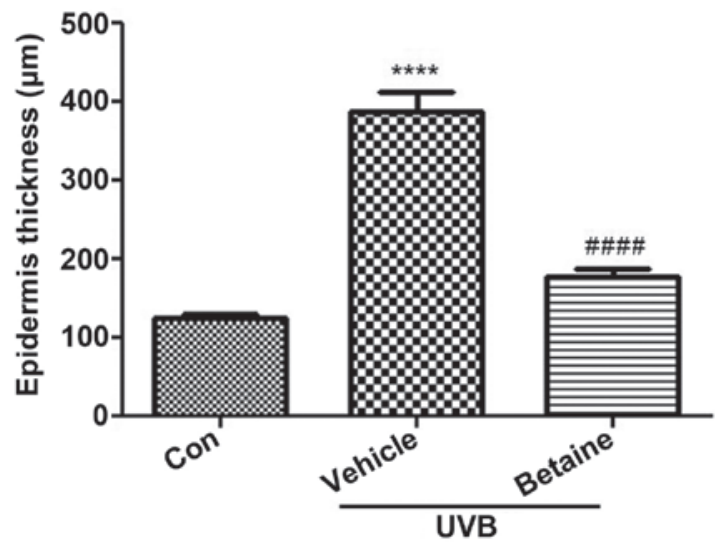

Figure 2. Betaine treatment decreases UVB-induced skin thickening in hairless mice. (A) Hematoxylin and eosin staining of UVB-irradiated hairless mice skin. (B) Quantification of epidermal thickness of dorsal skin. Original magnification, $\mathrm{x} 200 .{ }^{* * * *} \mathrm{P}<0.0001$, vs. Con. ${ }^{\# \# \# "} \mathrm{P}<0.0001$ vs. UVB/vehicle UVB, ultraviolet B; Con, control.

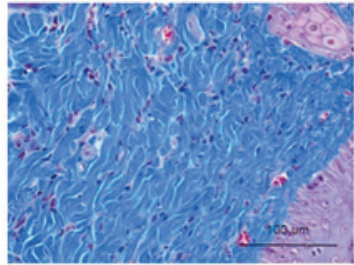

Control

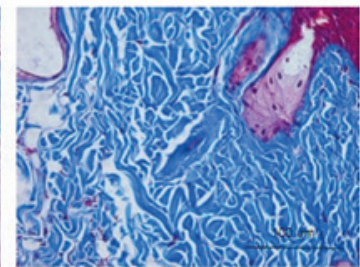

Vehicle

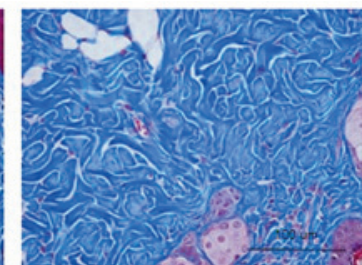

Betaine

UVB

Figure 3. Betaine has a protective effect on collagen fibers. Histological observation of hairless mice dermal tissue samples following Masson's trichrome stain. Collagen fibers were stained blue. Images were captured at magnification x200. UVB, ultraviolet B.

One-way analysis of variance and Turkey multiple comparisons test were used to analyze the results using Prism 5.0 software (GraphPad Software, Inc., La Jolla, CA, USA). P<0.05 was considered to indicate a statistically significant result.

\section{Results}

Betaine inhibits UVB-induced wrinkle formation. Wrinkle formation was observed in the dorsal region of hairless mice. UVB exposure induced skin wrinkles, however, treatment with betaine inhibited wrinkle formation (Fig. 1A). To evaluate the inhibitory activity of betaine on wrinkles, replicas of the dorsal skin were analyzed using an image analysis system to quantify the degree of wrinkle formation. The mean length and depth of the wrinkles in the UVB-treated groups were significantly increased, compared with those in the control group (Fig. 1B and $\mathrm{C}$ ). Treatment with betaine significantly reduced the mean length and depth of skin wrinkles. In addition, the number of wrinkles was also lower in the betaine-treated group, compared with the vehicle-treated group (Fig. 1D).

Betaine decreases the thickness of the epidermis in $U V B$-induced hairless mice. The effects of betaine on the changes in epidermal thickness in UVB-irradiated hairless mice were subsequently examined. Histological observation revealed that the thickness of the epidermis was significantly increased to $387.64 \mu \mathrm{m}$ following UVB irradiation, as shown by H\&E staining (Fig. 2A). Compared with the UVB-treated vehicle group, betaine treatment significantly inhibited the increase in epidermal thickness (176.94 $\mu \mathrm{m}$; Fig. 2B).

Betaine reduces collagen fiber damage in UVB-irradiated hairless mice. To visualize changes in collagen fibers in the dermal areas, histological sections of skin were subjected to Masson's trichrome staining. The collagen fibers were stained blue in the dermal areas (Fig. 3). Compared with the 
A
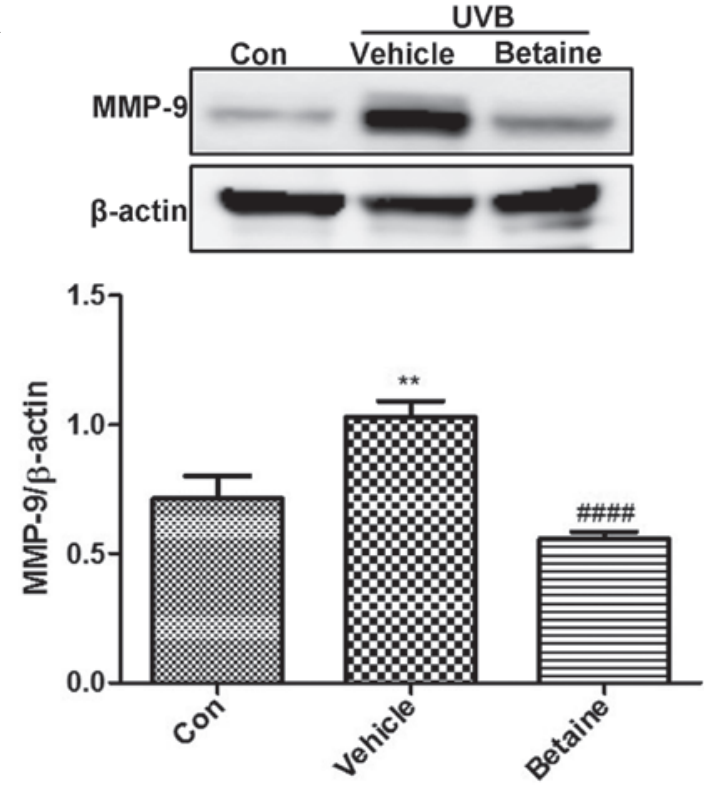

B
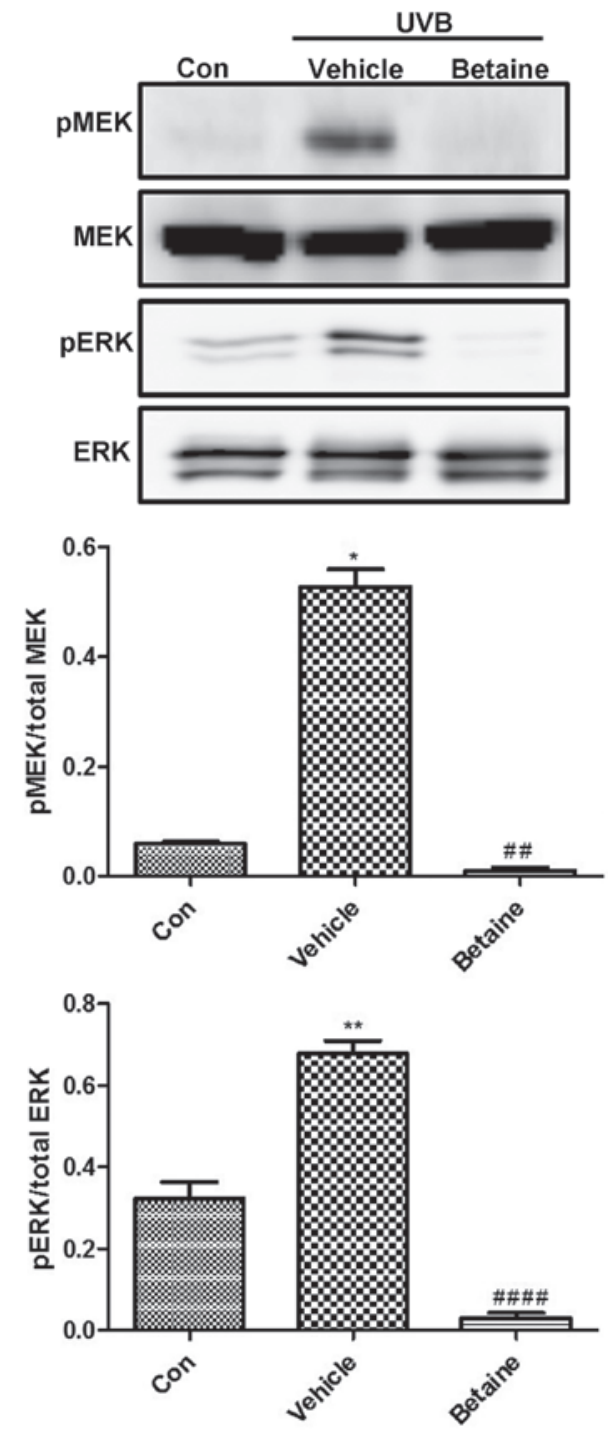

Figure 4. Betaine inhibits the expression of MMP- 9 and the phosophorylation of MEK and ERK. (A) Expression of MMP-9 and (B) phosphorylated (p) MEK and pERK. Proteins were extracted from murine dermal tissue samples, and expression levels were detected using western blot analysis. ${ }^{*} \mathrm{P}<0.05$, and ${ }^{* *} \mathrm{P}<0.01$ vs. Con. ${ }^{\# \#} \mathrm{P}<0.01$ and ${ }^{\# \# \# "} \mathrm{P}<0.0001$ vs. UVB/vehicle MMP-9, matrix metalloproteinase-9; MEK, mitogen-activated protein kinase kinase; ERK, extracellular signal-regulated kinase; UVB, ultraviolet B; Con. control. control group, the UVB-irradiated vehicle group exhibited a decrease in the abundance and density of collagen fibers. However, the collagen fibers in the betaine-treated group exhibited less collagen fiber damage, compared with those in the UVB-irradiated vehicle-treated mice.

Betaine inhibits the expression of MMP-9 and the phosphorylation of MEK and ERK in UVB-irradiated hairless mice. The effects of betaine on the expression and activity levels of several important modulators of photoaging were also investigated in the present study. UVB irradiation induced the expression of MMP-9 (Fig. 4A). However, betaine protected against UVB-induced photodamage by suppressing the expression of MMP-9. In addition, betaine inhibited the UVB-induced increase in the phosphorylation of MEK and ERK (Fig. 4B). These data indicated that betaine inhibited UVB-induced MEK and ERK activation.

\section{Discussion}

UV irradiation increases collagenase activity and reduces the production of collagen, resulting in wrinkle formation through the degradation of collagen in the dermal extracellular matrix (16). Exposure to UV light produces free radicals, releasing pro-inflammatory cytokines and growth factors, which activate proteases that degrade collagen elastin (17). The present study investigated the effects of betaine on UV irradiation-induced skin aging, particularly on the development of skin wrinkles, in a hairless mouse model.

Collagen and elastin protein fibers, the two main components of the dermis, act as a structural support system and provide the skin with strength and resilience (18). Following exposure to UV irradiation, dermal damage is predominantly manifested histologically as the disorganization of collagen fibrils and the accumulation of abnormal elastin-containing material (19). Exposure of the human skin to acute UV irradiation induces the expression of several MMPs, which degrade collagen fibrils and other components of the dermal extracellular matrix $(20,21)$. These pathological changes can lead to the formation of skin wrinkles $(22,23)$. In the mouse model used in the present study, UVB-irradiated murine skin exhibited increased wrinkle formation, and betaine inhibited this effect, suggesting that betaine may prevent UVB-associated collagen damage. Reduced damage to collagen fibers in the betaine-treated mice was observed, supporting this hypothesis.

MMP-9 is one of the primary enzymes associated with the degradation of skin collagen and components of the elastic fibers network. In addition, expression of MMP-9 in the epidermis has been reported to cause apoptosis, photoaging and inflammation by stimulating the expression of inflammatory cytokines, including as tumor necrosis factor- $\alpha$ and interleukin-1 $\beta$ (24). In the present study, western blot analysis demonstrated that betaine attenuated UVB-induced expression of MMP-9 regulated by the MEK/ERK pathway. MAPKs encompass serine/threonine kinases, which are involved in regulating several cellular processes, including proliferation, differentiation, stress adaptation and apoptosis (24). In addition to these functions, MAPKs are known to regulate the expression of MMP-9 (24). In a previous study, 
mangiferin isolated from Anemarrhena asphodeloides was shown to inhibit UVB-induced wrinkle formation and the expression of MMP-9 (25). Similarly, in the present study, betaine inhibited UVB-induced epidermal thickening and the protein expression of MMP-9. Therefore, betaine may exert its protective effects in a manner similar to that of mangiferin, by inhibiting the MAPK pathway, inhibiting the expression of MMP-9.

Epidermal thickness is used as a parameter to reflect quantitative changes in skin photoaging, as epidermal hypertrophy is considered to cause wrinkle formation (26). Furthermore, an increase in epidermal thickness occurs following UV exposure and assists in protecting the skin from further UV damage (27). In the present study, the epidermal thickness of the dorsal skin was increased by UVB exposure, however, this effect was significantly inhibited by betaine administration prior to UVB exposure. These data further supported that betaine protected the skin against UVB-induced damage.

In conclusion, the present study examined the anti-photoaging effects of betaine in a hairless mouse model of UVB-induced skin damage. The oral administration of betaine reduced the occurrence of characteristics associated with skin aging. Furthermore, betaine inhibited UVB-induced increases in skin thickness, wrinkle formation and collagen fiber loss in the hairless mice. These data demonstrated that these effects were mediated through a pathway involving MEK, ERK and MMP-9. Our results provide a evidence of the photoprotective effect of orally administered betaine, and suggest it may serve as a photoprotector against UVB-induced skin damage.

\section{Acknowledgements}

The present study was supported by a grant from the Korea Institute of Oriental Medicine (grant no. K14101).

\section{References}

1. Ganceviciene R, Liakou AI, Theodoridis A, Makrantonaki E and Zouboulis CC: Skin anti-aging strategies. Dermatoendocrinol 4: 308-319, 2012.

2. Tsatsou F, Trakatelli M, Patsatsi A, Kalokasidis K and Sotiriadis D: Extrinsic aging: UV mediated skin carcinogenesis. Dermatoendocrinol 4: 285-297, 2012.

3. Pyun HB, Kim M, Park J, Sakai Y, Numata N, Shin JY, Shin HJ, Kim DU and Hwang JK: Effects of collagen tripeptide supplement on photoaging and epidermal skin barrier in UVB-exposed hairless mice. Prev Nutr Food Sci 17: 245-253, 2012.

4. El-Domyati M, Attia S, Saleh F, Brown D, Birk DE, Gasparro F, Ahmad $\mathrm{H}$ and Uitto J: Intrinsic aging vs. photoaging: A comparative histopathological, immunohistochemical, and ultrastructural study of skin. Exp Dermatol 11: 398-405, 2002.

5. Afaq F and Mukhtar H: Botanical antioxidants in the prevention of photocarcinogenesis and photoaging. Exp Dermatol 15: 678-684, 2006

6. Zouboulis CC and Makrantonaki E: Clinical aspects and molecular diagnostics of skin aging. Clin Dermatol 29: 3-14, 2011.

7. Quan T, Qin Z, Xia W, Shao Y, Voorhees JJ and Fisher GJ. Matrix-degrading metalloproteinases in photoaging. J Investig Dermatol Symp Proc 14: 20-24, 2009.
8. Gelse K, Pöschl E and Aigner T: Collagens-structure, function and biosynthesis. Adv Drug Deliv Rev 55: 1531-1546, 2003.

9. Kim MS, Kim YK, Cho KH and Chung JH: Regulation of type I procollagen and MMP-1 expression after single or repeated exposure to infrared radiation in human skin. Mech Ageing Dev 127: 875-882, 2006.

10. Holvoet S, Vincent C, Schmitt D and Serres M: The inhibition of MAPK pathway is correlated with down-regulation of MMP-9 secretion induced by TNF-alpha in human keratinocytes. Exp Cell Res 290: 108-119, 2003.

11. Brenneisen P, Sies $H$ and Scharffetter-Kochanek K: Ultraviolet-B irradiation and matrix metalloproteinases: From induction via signaling to initial events. Ann NY Acad Sci 973: 31-43, 2002.

12. Shin YG, Cho KH, Kim JM, Park MK and Park JH: Determination of betaine in lycium chinense fruits by liquid chromatography-electrospray ionization mass spectrometry. J Chromatogr A 857: 331-335, 1999.

13. Cave M, Deaciuc I, Mendez C, Song Z, Joshi-Barve S, Barve S and McClain C: Nonalcoholic fatty liver disease: Predisposing factors and the role of nutrition. J Nutr Biochem 18: 184-195, 2007.

14. Bidulescu A, Chambless LE, Siega-Riz AM, Zeisel SH and Heiss G: Usual choline and betaine dietary intake and incident coronary heart disease: The atherosclerosis risk in communities (ARIC) study. BMC Cardiovasc Disord 7: 20, 2007.

15. Im AR, Kim YH, Uddin MR, Chae S, Lee HW, Kim YH, Kim YS and Lee MY: Betaine protects against rotenone-induced neurotoxicity in PC12 cells. Cell Mol Neurobiol 33: 625-635, 2013.

16. Chiang HM, Chen HC, Chiu HH, Chen CW, Wang SM and Wen KC: Neonauclea reticulata (Havil.) merr stimulates skin regeneration after UVB exposure via ROS scavenging and modulation of the MAPK/MMPs/collagen pathway. Evid Based Complement Alternat Med 2013: 324864, 2013.

17. Afaq F, Adhami VM and Mukhtar H: Photochemoprevention of ultraviolet B signaling and photocarcinogenesis. Mutat Res 571: 153-173, 2005.

18. Hou H, Li B, Zhang Z, Xue C, Yu G, Wang J, Bao Y, Bu L, Sun J, Peng Z and Su S: Moisture absorption and retention properties and activity in alleviating skin photodamage of collagen polypeptide from marine fish skin. Food Chem 135: 1432-1439, 2012

19. Vicentini FT, Fonseca YM, Pitol DL, Iyomasa MM, Bentley MV and Fonseca MJ: Evaluation of protective effect of a water-in-oil microemulsion incorporating quercetin against UVB-induced damage in hairless mice skin. J Pharm Pharm Sci 13: 274-285, 2010.

20. Vayalil PK, Mittal A, Hara Y, Elmets CA and Katiyar SK: Green tea polyphenols prevent ultraviolet light-induced oxidative damage and matrix metalloproteinases expression in mouse skin. J Invest Dermatol 122: 1480-1487, 2004

21. Ryu J, Park SJ, Kim IH, Choi YH and Nam TJ: Progective effect of porphyra-334 on UVA-induced photoaging in human skin fibroblasts. Int J Mol Med 34: 796-803, 2014.

22. Bosset S, Barré P, Chalon A, Kurfurst R, Bonté F, André P, Perrier P, Disant F, Le Varlet B and Nicolas JF: Skin ageing: Clinical and histopathologic study of permanent and reducible wrinkles. Eur J Dermatol 12: 247-252, 2002.

23. Chauhan $P$ and Shakya M: Modeling signaling pathways leading to wrinkle formation: Identification of the skin aging target. Indian J Dermatol Venereol Leprol 75: 463-468, 2009.

24. Onoue S, Kobayashi T, Takemoto Y, Sasaki I and Shinkai H: Induction of matrix metalloproteinase 9 secretion from human keratinocytes in culture by ultraviolet B irradiation. J Dermatol Sci 33: 105-111, 2003.

25. Kim HS, Song JH, Youn UJ, Hyun JW, Jeong WS, Lee MY, Choi HJ, Lee HK and Chae S: Inhibition of UVB-induced wrinkle formation and MMP-9 expression by mangiferin isolated from anemarrhena asphodeloids. Eur J Pharmacol 689: 38-44, 2012.

26. Urikura I, Sugawara T and Hirata T: Protective effect of fucoxanthin against UVB-induced skin photoaging in hairless mice. Biosci Biotechnol Biochem 75: 757-760, 2011.

27. Rabe JH, Mamelak AJ, McElgunn PJ, Morison WL and Sauder DN: Photoaging: Mechanisms and repair. J Am Acad Dermatol 55: 1-19, 2006. 